\title{
Qualidade de tomate 'Débora' minimamente processado armazenado em dois tipos de embalagens
}

\author{
Ana Carolina A Miguel; João Ricardo PS Dias; Marta Helena F Spoto; Roberta Teresa Rizzo-Benato \\ ESALQ/USP, Depto. Agroindústria, Alimentos e Nutrição, 13418-900 Piracicaba-SP; acmiguel@esalq.usp.br
}

\section{RESUMO}

Este trabalho teve como objetivo avaliar a qualidade de tomates cv. Débora minimamente processados acondicionados em dois tipos de embalagens. Os frutos foram lavados e sanificados com hipoclorito de sódio $\left(200 \mathrm{mg} \mathrm{L}^{-1}\right)$. A polpa destes frutos foi cortada em cubos $\left(1 \mathrm{~cm}^{3}\right)$ com posterior enxagüe em solução de hipoclorito de sódio $\left(20 \mathrm{mg} \mathrm{L}^{-1}\right)$, acondicionada em sacos de polipropileno (PP) ou copos de tereftalato de polietileno (PET) e armazenada a $5^{\circ} \mathrm{C}$ por 8 dias. Durante esse período foram retiradas diariamente amostras para determinação da perda de massa, coloração interna, textura, pH, conteúdos de sólidos solúveis (SS), acidez titulável (AT) e ácido ascórbico. Os teores de sólidos solúveis, acidez titulável, relação SS/AT e o $\mathrm{pH}$ se mostraram significativamente afetados pela embalagem. Os cubos acondicionados em copos PET apresentaram maiores teores de SS e menores teores de AT, o que resultou em uma relação SS/AT maior que os mantidos em sacos PP. O acondicionamento em copos PET mostrou-se o mais indicado para a produção de tomates cortados em cubos.

Palavras-chave: Lycopersicon esculentum, processamento mínimo, armazenamento.

\begin{abstract}
Quality of minimally processed tomato cv. Débora stored in two types of packages

The quality of minimally processed tomatoes cv. Débora conditioned in two types of packages was evaluated. The fruits were washed and sanitized with a $200 \mathrm{mg} \mathrm{L}^{-1}$ sodium hypochlorite solution. The pulp was cut in cubes $\left(1 \mathrm{~cm}^{3}\right)$, rinsed with a $20 \mathrm{mg} \mathrm{L}^{-1}$ sodium hypochlorite solution, packed in polypropylene (PP) bags or polyethylene terephthalate (PET) cups and stored at $5^{\circ} \mathrm{C}$ for eight days. During this period samples were daily retired to evaluate the loss of fresh mass, internal color, texture, $\mathrm{pH}$, contents of soluble solids (SS), titratable acidity (TA) and ascorbic acid. The soluble solids, titratable acidity, SS/TA ratio and $\mathrm{pH}$ were significantly affected by the packing type. The cubes packed in cups showed greater content of SS and lower of TA, resulting in a better ratio when compared to the cubes packed in bags. The polyethylene terephthalate (PET) cups were the best package for tomatoes cut in cubes.
\end{abstract}

Keywords: Lycopersicon esculentum, minimally processed, storage.

(Recebido para publicação em 25 de outubro de 2006; aceito em 12 de novembro de 2007)

$\mathrm{O}$ tomate é uma das olerícolas com maiores índices de perdas pós-colheita, decorrentes do manuseio inadequado nas etapas de colheita e transporte (Moura et al., 2005). Produtos hortícolas minimamente processados apresentam a vantagem de aliar conveniência, qualidade, redução de perdas e agregação de valor ao produto agrícola, possibilitando o aumento da competitividade pelo setor produtivo (Chitarra, 1998). Por outro lado, o processamento mínimo envolve fatores relevantes, tais como aceleração do metabolismo, degradação de pigmentos e vitaminas, além de escurecimento dos tecidos (Moretti et al., 2001), levando à redução da vida útil.

A comercialização de tomates minimamente processados poderia promover um incremento na demanda de tomates frescos e, por conseguinte, no seu consumo pelos mercados residencial e institucional, nos quais participa na composição de saladas (Mencarelli \& Saltveit, 1988).
Segundo Gil et al. (2002), o tomate se deteriora rapidamente após o corte, em comparação com outros vegetais, e cultivares 'longa-vida' têm sido destinadas ao processamento visando retardar o amadurecimento e prolongar a vida útil do produto.

Os maiores defeitos observados em tomate minimamente processado durante o processamento e o armazenamento são o acúmulo de líquido na embalagem e a condensação de água (Mencarelli et al., 1989; Gil et al., 1999).

A embalagem constitui-se um dos métodos disponíveis para controlar ou minimizar os processos degradativos, possibilitando que os produtos minimamente processados cheguem com qualidade aos consumidores (Wiley, 1997).

Estudos sobre as alterações que ocorrem em tomates minimamente processados são de extrema importância para o oferecimento de produtos de qualidade. Entretanto, a literatura a respeito de métodos para a manutenção da qualida- de de tomates minimamente processados é escassa (Artés et al., 1999). Assim, este trabalho teve como objetivo avaliar a qualidade de tomates 'Débora' minimamente processados, acondicionados em dois tipos de embalagem.

\section{MATERIAL E MÉTODOS}

Foram utilizados tomates 'Débora' no estádio de maturação break (coloração $50 \%$ verde e $50 \%$ vermelha) adquiridos no comércio de Piracicaba, SP. Os frutos foram lavados e desinfetados com hipoclorito de sódio $\left(200 \mathrm{mg} \mathrm{L}^{-1}\right)$ por 10 minutos. Foram retiradas manualmente as partes do pedúnculo e da polpa e os frutos foram cortados em cortador manual no formato de cubos $\left(1 \mathrm{~cm}^{3}\right)$, submetidos ao enxagüe em solução de hipoclorito de sódio (20 mg $\left.\mathrm{L}^{-1}\right)$ por 10 minutos, escorridos por $3 \mathrm{mi}-$ nutos, acondicionados em sacos de polipropileno (PP) $(18 \times 25 \times 0,10 \mathrm{~cm})$ ou acondicionados em copos Galvanni ${ }^{\circledR}$ 
50/35 $450 \mathrm{~mL}$, de tereftalato de polietileno (PET) munidos de tampa, contendo aproximadamente $150 \mathrm{~g}$ de pedaços, e armazenados sob refrigeração $\left(5,0 \pm 1,03^{\circ} \mathrm{C}\right.$ e $88,25 \%$ UR) por 8 dias. Durante este período foram retiradas amostras diariamente para determinação da perda de massa, mediante a pesagem em balança analítica; coloração interna determinada utilizando-se reflectômetro Minolta Chromameter CR 200b, através do sistema CIE 1976 (Minolta Corp, 1994) permitindo determinar a luminosidade $(L)$, o ângulo de cor $\left(h^{o}\right)$ e a cromaticidade $(C)$; textura determinada através da célula suculômetro do "Texture Test System" mod. TP-1; pH determinado em potenciômetro (AOAC, 1992); conteúdos de sólidos solúveis e de acidez titulável (AOAC, 1992) e ácido ascórbico (Strohecker \& Henning, 1967). O delineamento experimental foi em esquema fatorial ( $2 \times 8)$, com três repetições. Os resultados foram submetidos à análise de variância pelo teste $\mathrm{F}$ e comparação de média pelo teste $\mathrm{F}$ de Tukey (5\%).

\section{RESULTADOS E DISCUSSÃO}

A perda de massa fresca foi sempre maior nos copos que nos sacos plásticos, possivelmente devido à capacidade hermética das embalagens experimentadas. A pequena perda de massa, durante o período de armazenamento, pode ser atribuída à proteção conferida pelas embalagens, o que está de acordo com Sarantópoulos (1999) e Durigan (2000). Observou-se aumento linear da perda de massa com o avanço do período de armazenamento, o que pode ser atribuído à perda de umidade e de material de reserva pela transpiração e respiração, respectivamente. A perda de massa ao longo dos 8 dias foi pequena, porém durante o armazenamento esta perda pode resultar em prejuízo econômico, pois os tomates são vendidos por unidade de massa (Figura 1). Segundo Mencarelli \& Saltveit (1988), tomates minimamente processados são muito suscetíveis à perda de massa.

A coloração do produto é um dos principais parâmetros para a caracterização da qualidade, uma vez que os con-

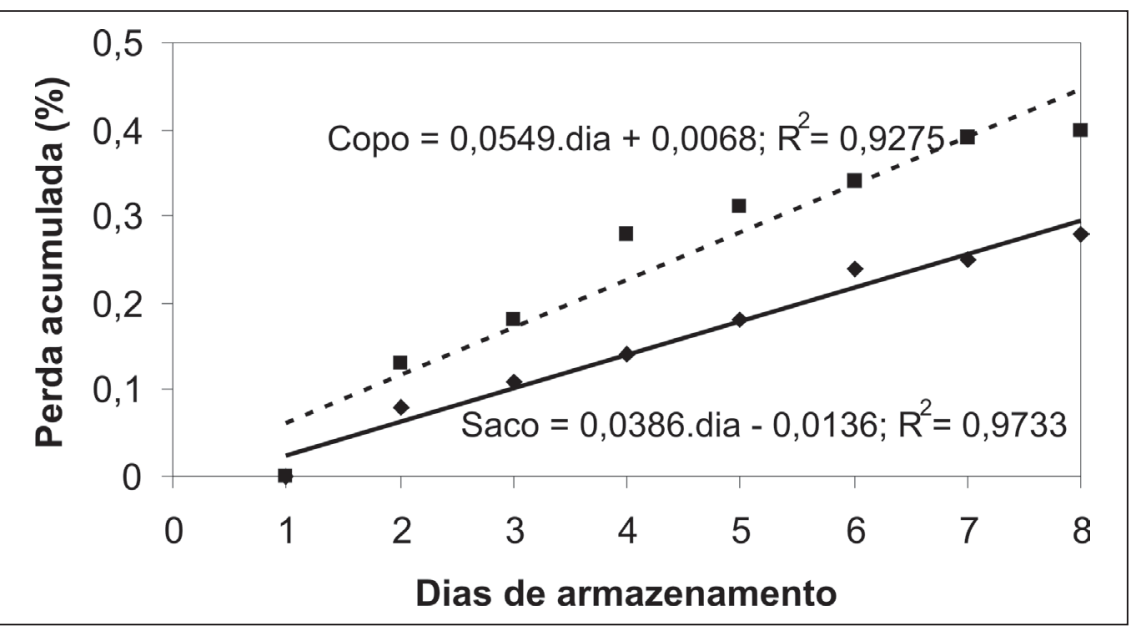

Figura 1. Evolução da perda de massa fresca de tomates 'Débora' minimamente processados e armazenados sob refrigeração $\left(5,0 \pm 1,03^{\circ} \mathrm{C}\right.$ e $88,25 \%$ UR) (evolution of fresh mass lose of tomatoes, cv. Débora minimally processed and stored under refrigeration $(5,0 \pm$ $1,03^{\circ} \mathrm{C}$ and 88,25\% relative umidity)). Piracicaba, ESALQ, 2006.

sumidores correlacionam esses dois fatores (Kays, 1991). Durante o armazenamento, observou-se um incremento nos valores de luminosidade (L) nos cubos de tomate acondicionados nos dois tipos de embalagens (Figura 2). O aumento neste atributo pode ser atribuído às perdas na coloração, frequientes em produtos minimamente processados (Durigan \& Cassaro, 2000).

O ângulo hue ou de cor mostrou-se variável ao longo do período de armazenamento, nos dois tipos de embalagens (Figura 2). Observou-se que os pedaços de tomate se apresentaram menos vermelhos, ou com maiores valores para o ângulo hue, passando de $45,8^{\circ} \mathrm{e}$ $45,63^{\circ}$ para $55,09^{\circ}$ e $51,62^{\circ}$, nos sacos de PP e nos copos, respectivamente. Segundo Shewfelt et al. (1988), a redução na coloração vermelha pode estar relacionada com o efeito do corte, o qual pode causar a perda do licopeno.

Para a cromaticidade, observou-se tendência de aumento nos valores, nas duas embalagens utilizadas, indicando o aumento de intensidade durante o período de armazenamento (Figura 2). Verificou-se que os cubos acondicionados em sacos de PP apresentaram coloração mais intensa em relação aos mantidos em copos no $6^{\circ}$ e no $8^{\circ}$ dia de armazenamento.

Os teores de ácido ascórbico se comportaram de forma equivalente nos dois tipos de embalagens testadas $(16,45$ 17,34 mg ácido ascórbico $100 \mathrm{~g}^{-1}$ ) e não foram significativamente afetados pelo período de armazenamento. Os cuidados tomados durante o processamento contribuíram para a manutenção desses teores, evitando a ocorrência de reações oxidativas, responsáveis pelo escurecimento (Wiley, 1997). Souza et al. (2005) verificaram redução nos teores de ácido ascórbico em tomates verdes minimamente processados, após 6 dias de armazenamento a $5^{\circ} \mathrm{C}$. Moura (1999) afirma que existe pouca variação no conteúdo de ácido ascórbico com a maturação dos frutos.

Durante o período de armazenamento, observou-se redução significativa na textura da polpa, de 9,53 $\mathrm{N}$ para 7,50 N, evento comumente encontrado para outras hortaliças e que tem sido atribuído à modificação nas estruturas e na composição da parede celular devido à ação de enzimas, conforme o indicado por Chitarra (1999). Apesar de a textura ter se modificado durante o armazenamento, os cubos acondicionados em PP $(8,62 \mathrm{~N})$ apresentaram resistência semelhante à dos mantidos em copos $(8,51 \mathrm{~N})$. Estes resultados estão de acordo com o observado por Artés et al. (1999), onde o período de armazenamento influenciou na textura de tomates minimamente processados.

Verificaram-se diferenças significativas nos teores de sólidos solúveis de tomates minimamente processados acondicionados nos dois tipos de embalagens, sendo que os cubos conserva- 


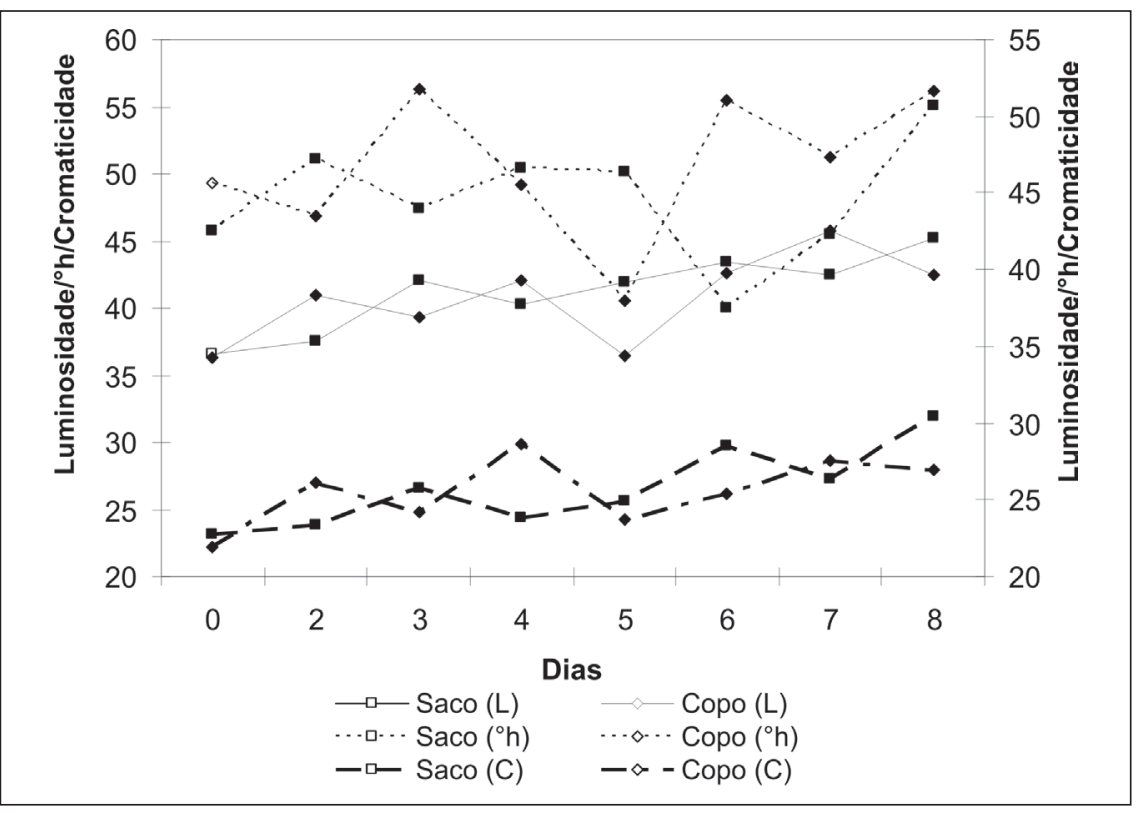

Figura 2. Evolução da coloração externa de tomates 'Débora' minimamente processados e armazenados sob refrigeração $\left(5,0^{\circ} \mathrm{C} \pm 1,03^{\circ} \mathrm{C}\right.$ e $88,25 \%$ UR) (evolution of the external coloration of 'Débora' tomatoes, minimally processed and stored under refrigeration $(5,0 \pm$ $1,03^{\circ} \mathrm{C}$ and 88,25\% relative umidity)). Piracicaba, ESALQ, 2006.

dos em PP $\left(4,32^{\circ}\right.$ Brix $)$ apresentaram valores inferiores aos acondicionados em copos $\left(4,62^{\circ}\right.$ Brix). Houve uma redução significativa nos teores de açúcar com o tempo de armazenamento, de 4,21 ${ }^{\circ}$ Brix para $3,94{ }^{\circ}$ Brix. Este decréscimo pode ser devido ao efeito da atividade metabólica intensificada pelas injúrias ocasionadas pelas operações de processamento, o que pode ser considerado um fator que atua negativamente na qualidade (Chitarra \& Chitarra, 1990). Os teores de sólidos solúveis verificados neste trabalho variaram de $3,94{ }^{\circ}$ Brix a $4,72{ }^{\circ}$ Brix e foram inferiores aos encontrados em tomates 'Durinta' minimamente processados (4,70-5,40 ${ }^{\circ}$ Brix) por Gil et al. (2002).

A variação nos valores médios de $\mathrm{pH}$ do suco (de 4,09 a 4,38) indica que os cubos acondicionados em sacos plásti$\cos (\mathrm{pH}=4,09)$ apenas diferiram daqueles mantidos em copos no último dia de armazenamento, sendo que os copos apresentaram maior valor médio $(\mathrm{pH}$ $=4,35$ ). Analisando a interação dos efeitos embalagem e período de armazenamento, verifica-se que os tomates mantidos em sacos mostraram diminuição com o avanço dos dias, passando de 4,28 para 4,09, diferentemente dos conservados em copos que não apresentaram variações significativas para este parâmetro, ou seja, mantivedos diferem do observado por Souza et al. (2005), que não verificaram diferenças significativas nos valores de $\mathrm{pH}$ em tomates verdes e maduros minimamente processados, armazenados a $5^{\circ} \mathrm{C}$ por 8 dias.

Os fatores embalagem e tempo de armazenamento, bem como sua interação, promoveram efeitos significativos nos valores da acidez titulável. Os teores de ácido cítrico mantiveramse estáveis durante o período de armazenamento nos tomates acondicionados em copos (0,18 a 0,23 g ácido cítrico $100 \mathrm{~g}^{-1}$ ), enquanto que os mantidos em sacos de PP apresentaram aumento na acidez titulável apenas no último dia de avaliação, passando de 0,23 $\mathrm{g}$ ácido cítrico $100 \mathrm{~g}^{-1}$ no $7^{\circ}$ dia para 0,35 $\mathrm{g}$ ácido cítrico $100 \mathrm{~g}^{-1}$. O conteúdo de ácidos orgânicos manteve-se estável até o $5^{\circ}$ dia de armazenamento, independente da embalagem. Estes resultados diferem do verificado por Gil et al. (2002) que constataram decréscimo na acidez titulável em tomates minimamente processados armazenados a $5^{\circ} \mathrm{C}$. A partir do $5^{\circ}$ dia, os cubos acondicionados em sacos plásticos apresentaram maior aci$\operatorname{dez}\left(0,24 \mathrm{~g}\right.$ ácido cítrico $\left.100 \mathrm{~g}^{-1}\right)$ que os cubos mantidos em copos $(0,20 \mathrm{~g}$ ácido ram-se entre 4,23 e 4,38. Estes resulta- cítrico $100 \mathrm{~g}^{-1}$ ). Este comportamento crescente pode estar relacionado ao processo fermentativo ocorrido com o avanço do período de armazenamento (Wiley, 1997).

A relação entre os teores de sólidos solúveis e de acidez titulável (SS/AT) foi influenciada pelo tipo de embalagem e pelo período de armazenamento, que variou de 11,88 a 24,85 (saco) e de 18,94 a 25,23 (copo). O maior teor de SS e a menor AT fez com que os cubos acondicionados em copos apresentassem as maiores relações SS/AT, a partir do terceiro dia. Com o avanço dos dias, os tomates mantidos em PP apresentaram decréscimo na relação SS/AT, como conseqüência do aumento nos seus teores de acidez, enquanto que os mantidos em copos apresentaram equilíbrio entre os teores de açúcares e ácidos. Essa relação é um importante atributo qualitativo, uma vez que indica o sabor inerente ao produto, o qual é resultado da contribuição dos componentes responsáveis pela acidez e doçura (Mattiuz, 2002). Segundo Gil et al. (2002), a relação SS/AT ótima para o consumo de tomate é de 14,5, sendo menor que os valores observados para o material testado neste trabalho, indicando que os produtos apresentaram boa qualidade, exceto para os tomates acondicionados em PP no $8^{\circ}$ dia de armazenamento, que apresentaram o valor de 11,88 para a relação SS/AT, resultante da maior contribuição dos ácidos. O equilíbrio entre os teores de açúcares e ácidos para os tomates mantidos em copos, de acordo com Carvalho \& Chitarra (1984), permite inferir que estes apresentaram manutenção da qualidade organoléptica, enquanto que os acondicionados em PP mostraram perda de qualidade.

$\mathrm{O}$ acondicionamento em copos PET mostrou-se o mais indicado para a conservação de tomates cortados em cubos.

\section{REFERÊNCIAS}

AOAC. 1992. Official methods of analysis of the Association of Analytical Chemists International. Washington: A.O.A.C. 1141p. ARTÉS F; CONESA MA; HERNÁNDEZ S; GIL MI. 1999. Keeping quality of fresh-cut tomato. Postharvest Biology and Technology 17: 153162. 
CARVALHO VD; CHITARRA MI. 1984. Aspectos qualitativos da uva. Informe Agropecuário 10: 75-79

CHITARRA MIF. 1998. Processamento mínimo de frutas e hortaliças. Viçosa: Centro de Produções Técnicas. 88p.

CHITARRA MI. 1999. Alterações bioquímicas do tecido vegetal com o processamento mínimo. In: SEMINÁRIO SOBRE HORTALIÇAS MINIMAMENTE PROCESSADAS. Palestra... Piracicaba: ESALQ - USP. 9p.

CHITARRA MIF; CHITARRA AB. 1990. Póscolheita de frutos e hortaliças: fisiologia e manuseio. Lavras: ESAL/FAEPE. 320p.

DURIGAN JF. 2000. O processamento mínimo de frutas. In: CONGRESSO BRASILEIRO DE FRUTICULTURA, 16. Palestra... Fortaleza: SBF. 12p.

DURIGAN JF; CASSARO KP. 2000. Hortaliças minimamente processadas. Horticultura Brasileira 18: 159-161.

GIL MI; CONESSA MA; ARTÉS F. 2002. Quality changes in fresh cut tomato as affected by modified atmosphere packaging. Postharvest Biology and Technology 25: 199-207.

GIL MI; HERNÁNDEZ S; CONESA MA; ARTÉS F. 1999. Keeping quality in minimally processed tomato slices by chemical treatments. Actas de Horticultura 4: 274-279.
KAYS SJ. 1991. Postharvest physiology of perishable plants products. New York: Van Nostrand Reinhold. 532p.

MATTIUZ BH. 2002. Efeitos de injúrias mecânicas e do processamento mínimo na fisiologia pós-colheita de goiabas. Jaboticabal: UNESP - FCAV. 120p (Tese doutorado).

MENCARELLI F; SALTVEIT ME. 1988. Ripening of mature-green tomato fruit slices. Journal of the American Society Horticultural Science 113: 742-745.

MENCARELLI F; SALTVEIT ME; MASSANTINI R. 1989. Lightly processed foods: ripening of tomato fruit slices. Acta Horticulturae 244: 193-200.

MINOLTA CORP. 1994. Precise color communication: color control from feeling to instrumentation. Ramsey: Minolta Corporation Instrument Systems Division. 49p.

MORETTI CL. 2001. Technology of fresh-cut products. In: BRAZILIAN CONGRESS OF AGRICULTURAL ENGINNEERING, 3. Anais... Foz do Iguaçu: SBEA. p. 357-364.

MOURA MA. 1999. Crescimento e pós-colheita de frutos do tomateiro cv. Santa Clara e do seu mutante firme. Viçosa: UFV. 86p (Tese doutorado).
MOURA ML; FINGER FL; MIZOBUTSI GP; GALVÃO HL. 2005. Fisiologia do amadurecimento na planta do tomate 'Santa Clara' e do mutante 'Firme'. Horticultura Brasileira 23: 81-85.

SARANTÓPOULOS CIGL. 1999. Embalagens para vegetais minimamente processados fresh-cut. In: SEMINÁRIO SOBRE HORTALIÇAS MINIMAMENTE PROCESSADAS. Palestra... Piracicaba: ESALQ - USP. 6p.

SHEWFELT RL; THAI CN; DAVIS JW. 1988. Prediction of changes in color of tomatoes during ripening at different constant temperatures. Journal of Food Science 53: 1433-1437.

STROHECKER R; HENNING HM. 1967. Analisis de vitaminas: métodos comprobados. Madrid: Paz Montalvo. 428p.

SOUZA JF; VIEIRA ACA; YAGUIU P; CARNELOSSI MA; JALALI VRR. 2005. Influência do estádio de maturação na conservação de tomate minimamente processado. Horticultura Brasileira 23:445.

WILEY RC. 1997. Frutas y hortalizas mínimamente procesadas y refrigeradas. Zaragoza: Acribia. 362p. 